\title{
Optimization of Cutoff Frequency Limits of Microstrip Circular Patch Antenna Printed on Ferrite Substrate by Artificial Neural Network Analysis
}

\author{
${ }^{1}$ Naveen Kumar Saxena, Raj Kumar Verma ${ }^{2}$, Nitendar Kumar ${ }^{3}$, P.K.S. Pourush ${ }^{4}$ \\ ${ }^{1}$ Microwave Lab, Department of Physics, Agra College Agra, PIN 282002, Agra (U.P), India. \\ ${ }^{2}$ Microwave Lab, Department of Physics, Agra College Agra, PIN 282002, Agra (U.P), India. \\ ${ }^{3}$ Solid State Physics Laboratory, Timarpur, Delhi 110007, India. \\ ${ }^{4}$ Microwave Lab, Department of Physics, Agra College Agra, PIN 282002, Agra (U.P), India.
}

\begin{abstract}
General artificial neural network (ANN) analysis has been applied to optimize the lower and upper frequency limits of cutoff region of switchable microstrip circular patch antenna printed on ferrite (LiTiZn) substrate in the X-band range. The ferrite substrate offered numbers of novel magnetic and electrical characteristics including switchable and polarized radiations from a microstrip antenna with DC magnetic biasing. For particular frequency limits most of the power will be converted into mechanical waves and very little radiates into air. Under such condition the antenna become switch off, in the sense of effectively absence as radiator. In this work Radial Basis Function (RBF) networks has been applied in ANN models. Synthesis has been defined as the forward side and then analysis as the reverse side of the problem. In the RBF network, the spread value has been chosen as 0.01 , which gives the best accuracy. RBF has been tested with 100 samples frequencies but trained only for particular cutoff 15 samples frequencies. The obtained optimized of value lower and upper cutoff frequency $\left(w_{l} \& w_{u}\right)$ are very close to the values measured through the material dispersion graph which has been plotted for perpendicular applied 1500 Oe magnetic field.
\end{abstract}

Key Words: Microstrip antennas, tunability, ferrite material, artificial neural networks, reverse modeling.

\section{Introduction}

In recent years magnetic materials have received much attention in high frequency applications. Ferrite is one of the important magnetic materials which are very useful in microwave antennas and devices. The ferrite offered number of novel magnetic and electrical characteristics including switchable and polarized radiations from a microstrip antenna with DC magnetic biasing.

Nonreciprocal behavior of ferrite substrate, on applying magnetic biasing tends to ordinary and extraordinary wave propagation. For particular frequency limit, most of the power in the form of extraordinary wave resonance with lattice magnetic vibrations and converted into mechanical waves and little radiates into air. Under such condition the antenna become switch off, in the sense of effectively absent as radiator [1-3]. In the literature, artificial neural network (ANN) models have been built usually for the analysis of microstrip antennas for circular and equilateral triangle patch geometries for computing their resonant frequencies on given dielectric substrate.

There are two popular learning algorithms for neural network implementation. These are the backpropagation algorithm and radial basis function algorithm. The backpropagation network is well known among the current types of neural network system available. It is a multilayer feed forward network with a different transfer function in the neuron and a more powerful learning rule. The learning rule is known as backpropagation, which is a kind of gradient decent technique with backward error propagation. The radial basis function (RBF) network is a two-layer network whose output units form a linear combination of the basis function is computed by the hidden unit. In the aspect of learning, the RBF network is much faster than the back propagation network. The primary reason for this is that the learning process in RBF network has two stages and both stages can be made efficient by using appropriate learning algorithms.

However, in the present work, for the synthesis we include ordinary wave propagation constant $\left(K_{d}\right)$, desired cutoff frequency $(w)$, applied magnetic frequency $\left(w_{o}\right)$ and internal magnetic frequency $\left(w_{m}\right)$ as input parameters to get the extraordinary wave propagation constant $\left(K_{e}\right)$ which is mainly responsible for the switchability of antenna. For analysis we include ordinary wave propagation constant $\left(K_{d}\right)$, extraordinary wave propagation constant $\left(K_{e}\right)$, applied magnetic frequency $\left(w_{o}\right)$, and internal magnetic frequency $\left(w_{m}\right)$ as input parameters to get the desired cutoff frequency limits $\left(w_{l} \& w_{u}\right)$ which is desirable and should be very precise for any communication system [4-9]. 


\section{MICROSTRIP ANTENNA}

The circular microstrip antennas with a circular patch of radius ' $a_{e}=5.3 \mathrm{~mm}$ ' over a ground plane with a LiTiZn ferrite substrate thickness ' $h=2 \mathrm{~mm}$ ' and dielectric constant ' $\varepsilon_{r}=16$ ' as shown in Fig.1 [10].

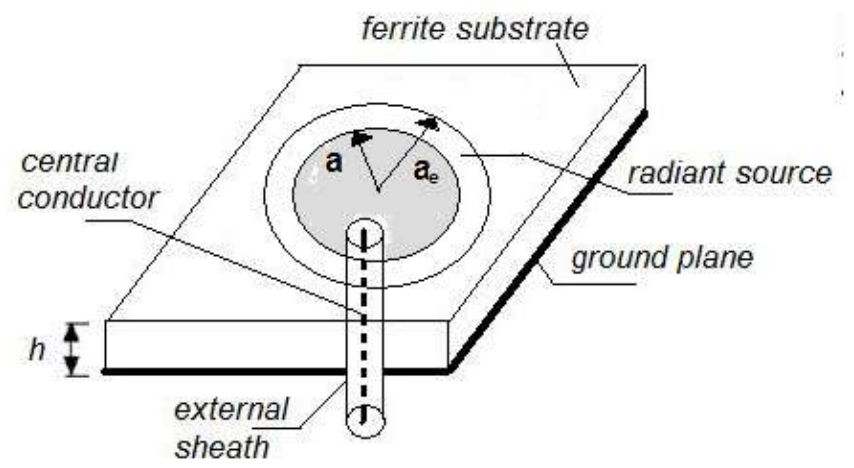

Fig. 1: Schematic Diagram of Microstrip Circular Patch Antenna

It has been established that, for a biased ferrite slab, a normal incident plane wave may excite two types of waves (ordinary and extraordinary wave). In the case of normal incident magnetic field biasing ordinary wave is same as the plane wave in the dielectric slab. On the other hand, the extraordinary wave is a TE mode polarized parallel to the biasing direction with its phase propagation constant $\mathrm{K}_{\mathrm{e}}[11]$.

$$
K_{e}=K_{d}\left[\frac{\left(w_{o}+w_{m}\right)^{2}-w^{2}}{w_{o}\left(w_{o}+w_{m}\right)-w^{2}}\right]^{1 / 2}
$$

Upper cutoff frequency limit

$$
w_{u}=\left(w_{o}+w_{m}\right)
$$

Lower cutoff frequency limit

where

$$
w_{l}=\left[w_{o}\left(w_{o}+w_{m}\right)\right]^{1 / 2}
$$

$$
\begin{gathered}
K_{d}=\frac{w}{c} \sqrt{\epsilon_{e f f}} \\
w=2 \pi f, w_{o}=\gamma H_{o} \text { and } w_{m}=\gamma 4 \pi M_{s}
\end{gathered}
$$

where $H_{o}$ is the bias field, $4 \pi M_{s}$ is the saturation magnetization, $\gamma$ is the gyromagnetic ratio as $\gamma=$ 2.8 MHz./Oe. Polycrystalline LiTiZn ferrite have been synthesized by the solid state reaction technique (SSRT).

Table 1: The electrical and magnetic properties of LiTiZn ferrite substrate

\begin{tabular}{|l|l|}
\hline \multicolumn{1}{|c|}{ LiTiZn Ferrite Characteristics } & \multicolumn{1}{c|}{ Values } \\
\hline Magnetic Saturation $\left(4 \pi M_{s}\right)$ & 2200 Gauss \\
\hline Curie Temperature $\left(\mathrm{T}_{\mathrm{c}}\right)$ & $385 \mathrm{~K}$ \\
\hline Density $(\rho)$ & 4.21 grams $/ \mathrm{cm}^{3}$ \\
\hline Remanence & 0.90 \\
\hline Coercivity & 1.50 \\
\hline Dielectric Constant $(\varepsilon)$ & 16 \\
\hline Resonance Line Width $(\Delta \mathrm{H})$ & 370 Oersteds \\
\hline Loss Tangent $(\tan \delta)$ & $<0.0005$ \\
\hline
\end{tabular}

The ingredients required for the preparation of these ferrites have been calculated on the basis of chemical formula.

$$
\mathrm{Li}_{0.63} \mathrm{Zn}_{0.1} \mathrm{Mn}_{0.1} \mathrm{Ti}_{0.36} \mathrm{Fe}_{1.81} \mathrm{O}_{4}
$$


Lithium ferrite is one of the most versatile magnetic materials. It is generally useful for microwave devices, memory-core, power-transformers in electronics, antennas, read/write heads for high speed digital tapes. This material is strategically important due to its high resistivity, low electric losses, high Curie temperature. The electrical and magnetic properties of LiTiZn ferrite substrate has been experimentally calculated in laboratory which is listed in table 1 .

\section{Neural Network Analysis}

A Radial Basis Function (RBF) network has been applied in ANN models and the synthesis / analysis problem to solve the electromagnetic formulae of the microstrip antennas. There are 2 points are especially emphasized: the extraordinary propagation constant $\left(\mathrm{K}_{\mathrm{e}}\right)$ which is mainly responsible for the switchability of antenna and the corresponding particular desired cutoff frequency $(w)$ which is very desirable to switch off the antenna system $[12,13]$. In the Forward Side of the problem the input/output quantities to the ANN synthesis and ANN analysis are shown in fig. 2.

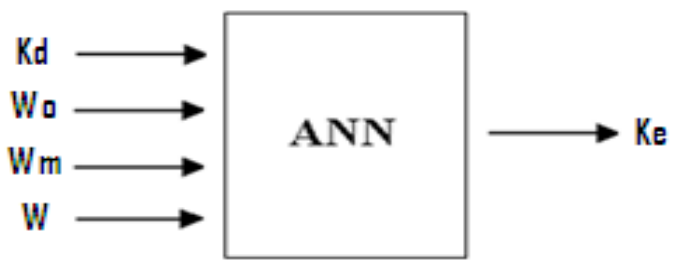

Fig. 2 Forward side ANN model.

In the analysis side of the problem, terminology similar to that in the synthesis mechanism is used, but the cutoff frequency of antenna is obtained from the output for a optimize extraordinary propagation constant, ordinary wave propagation constant $\left(\mathrm{K}_{\mathrm{d}}\right)$, applied magnetic frequency $\left(\mathrm{w}_{\mathrm{o}}\right)$, and internal magnetic frequency $\left(\mathrm{w}_{\mathrm{m}}\right)$. In the Reverse Side of problem the feed forward neural networks with a single hidden layer that use radial basis activation functions for hidden neurons are called radial basis function networks. RBF networks are applied for various microwave modeling purposes. A typical RBF network structure is given in Fig. 3.

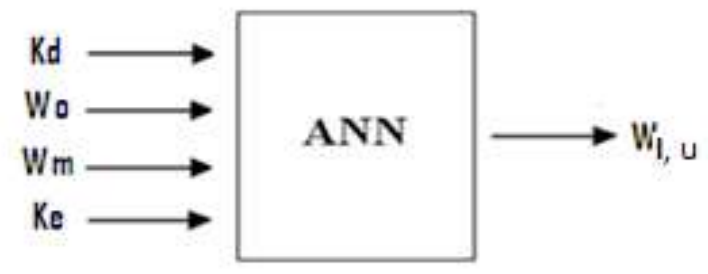

Fig. 3 Reverse side ANN model.

The parameters $c_{i j}$ and $\lambda_{i j}$ are centers and standard deviations of radial basis activation functions. Commonly used radial basis activation functions are Gaussian and multi-quadratic. Given the inputs $\mathrm{x}$, the total input to the $i^{\text {th }}$ hidden neuron $Y_{i}$ is given by

$$
\gamma_{i}=\sqrt{\sum_{j=1}^{n}\left(\frac{x_{j}-c_{i j}}{\lambda_{i j}}\right)^{2}}, i=1,2,3 \ldots \ldots . N
$$

where $\mathrm{N}$ is the number of hidden neurons.

In the RBF network, the spread value was chosen as 0.01 , which gives the best accuracy. RBF is trained with 100 samples frequencies but tested only for particular cutoff 15 samples frequencies. 4 inputs and 1 output were used for both the analysis ANN the synthesis ANN. The training and test data of the synthesis and analysis ANN were obtained from both experimental results given in previous works and a computer program using formulae given in previous section.

\section{Performance And Result}

For synthesis and analysis, RBF network provide the best approximation to the tabulated target values depicted from tables 2 and 3 respectively. The performance, of synthesis ANN is 0.000389852 at 79 epochs while that of analysis ANN is 0.00092708 at 36 epochs \& 0.000585216 at 34 epochs. The results of the synthesis and analysis ANN for ferrite material can be compare with dispersion graph (fig. 7) obtained for ferrite 
material. Switchability and tunability regions are clearly shown in the dispersion curve plotted at 1500 Oe for LiTiZn-ferrite slab, on which circular microstrip patch antenna has been designed.. It is important to mention that switchability region is of great concern for the present analysis. From the graph and table $\left(w_{l} \& w_{u}\right)$, we can conclude and compare the optimal accuracy for the synthesized and optimized extraordinary propagation constant $\left(\mathrm{K}_{\mathrm{e}}\right)$. The optimized value of $\mathrm{K}_{\mathrm{e}}$ is around 0.3898 and of lower cutoff frequency $\mathrm{w}_{1}=0.5852 \mathrm{GHz} \&$ of upper cutoff frequency $\mathrm{w}_{\mathrm{u}}=0.9270 \mathrm{GHz}$. These optimized value are very close to cutoff values depicted in dispersion graph.

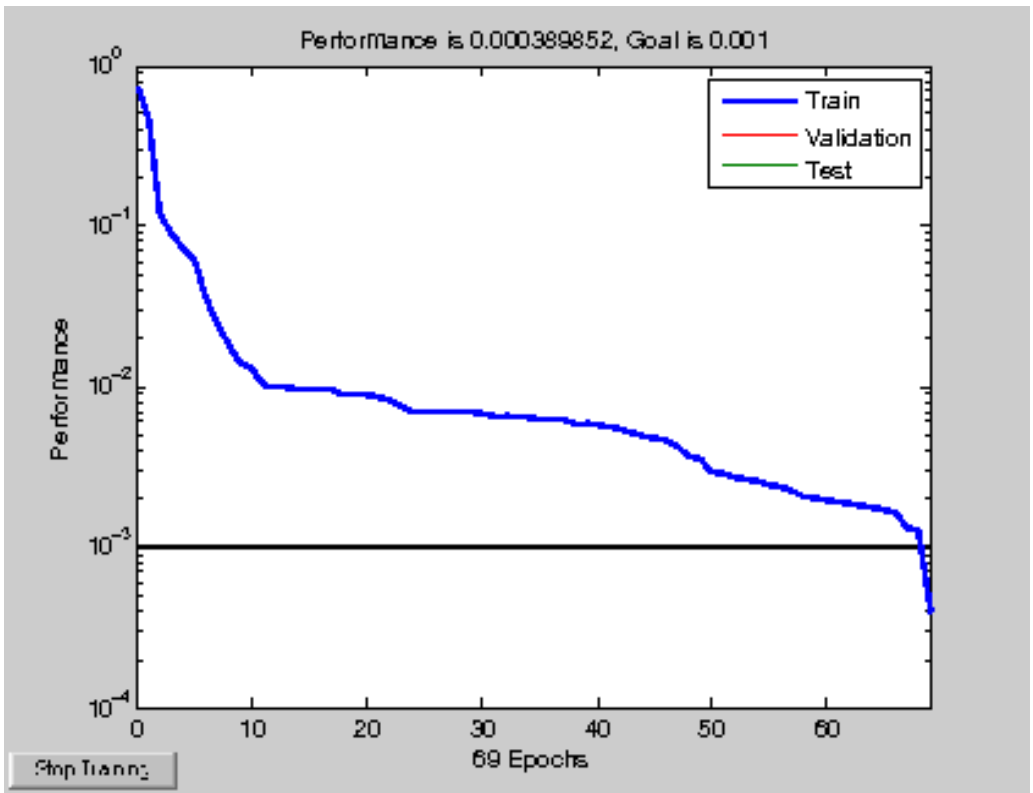

Fig. 4 Training Response for synthesis at 69 Epochs

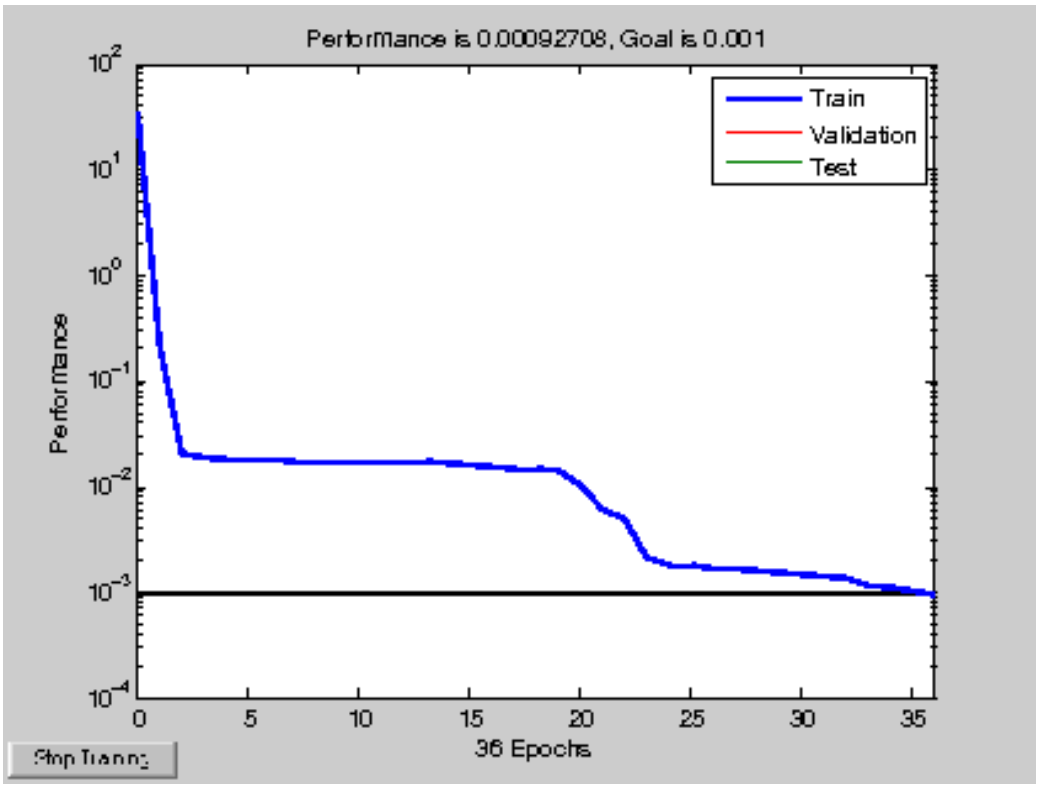

Fig. 5 Training Response for lower frequency limit at 36 Epochs 


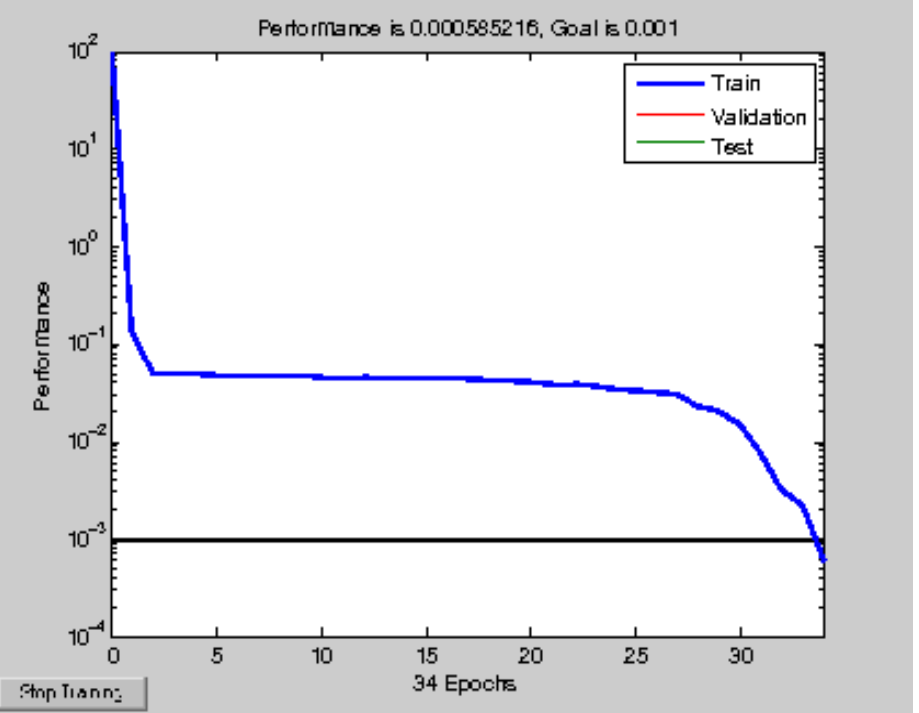

Fig. 6 Training Response for upper frequency limit at 34 Epochs

Table 2: Results of the synthesis ANN and comparison with target

\begin{tabular}{|c|c|c|c|c|c|}
\hline $\boldsymbol{K}_{\boldsymbol{d}}$ & $\boldsymbol{w}_{\boldsymbol{o}}(\mathbf{G H z})$ & $\boldsymbol{w}_{\boldsymbol{m}}(\mathbf{G H z})$ & $\boldsymbol{w}(\mathbf{G H z})$ & $\boldsymbol{K}_{\boldsymbol{e}}$-target & $\boldsymbol{K}_{\boldsymbol{e}}$-RBF \\
\hline 8.9630 & 4.2000 & 6.1575 & 6.4277 & 0.3335 & 0.32732 \\
\hline 8.7002 & 4.3400 & 5.9816 & 6.2392 & 0.9835 & 0.95907 \\
\hline 8.6125 & 4.3680 & 6.0168 & 6.1764 & 0.9771 & 0.94611 \\
\hline 9.1996 & 4.2896 & 6.0520 & 6.5973 & 0.5429 & 0.53367 \\
\hline 7.2720 & 4.2560 & 5.8408 & 5.2150 & 0.1858 & 0.22136 \\
\hline 8.0868 & 4.2280 & 6.2279 & 5.7994 & 0.4066 & 0.38036 \\
\hline 8.1745 & 4.3120 & 6.3335 & 5.8622 & 0.3808 & 0.3929 \\
\hline 8.9630 & 4.3120 & 6.2631 & 6.4277 & 0.3646 & 0.35101 \\
\hline 9.4011 & 4.2616 & 6.2983 & 6.7419 & 0.3105 & 0.30621 \\
\hline 8.1307 & 4.2308 & 5.8408 & 5.8308 & 0.6756 & 0.67532 \\
\hline 7.8643 & 4.2336 & 5.9464 & 5.7994 & 0.9988 & 0.99136 \\
\hline 9.3979 & 4.2364 & 6.1223 & 6.9304 & 0.2860 & 0.30701 \\
\hline 9.5428 & 4.2700 & 6.0871 & 7.0372 & 0.3294 & 0.30783 \\
\hline 6.7566 & 4.3848 & 6.3335 & 4.9826 & 0.5779 & 0.58706 \\
\hline 6.3875 & 4.3540 & 6.1927 & 4.5742 & 0.9913 & 0.95661 \\
\hline 8.6354 & 4.6368 & 6.3335 & 6.2832 & 0.6984 & 0.69622 \\
\hline
\end{tabular}

Table 3: Results of the analysis ANN and comparison with target

\begin{tabular}{|c|c|c|c|c|c|c|c|}
\hline $\boldsymbol{K}_{\boldsymbol{d}}$ & $\begin{array}{c}\boldsymbol{w}_{\boldsymbol{o}} \\
(\mathbf{G H z})\end{array}$ & $\begin{array}{c}\boldsymbol{w}_{\boldsymbol{m}} \\
(\mathbf{G H z})\end{array}$ & $\boldsymbol{K}_{\boldsymbol{e}}$ & $\begin{array}{c}\boldsymbol{w}_{\boldsymbol{l}}-\text { target } \\
(\mathbf{G H z})\end{array}$ & $\begin{array}{c}\boldsymbol{w}_{\boldsymbol{l}} \mathbf{- R B F} \\
(\mathbf{G H z})\end{array}$ & $\begin{array}{c}\boldsymbol{w}_{\boldsymbol{u}}-\text { target } \\
(\mathbf{G H z})\end{array}$ & $\begin{array}{c}\boldsymbol{w}_{\boldsymbol{u}}-\mathbf{R B F} \\
(\mathbf{G H z})\end{array}$ \\
\hline 8.0000 & 4.2000 & 6.1575 & 0.3273 & 6.5956 & 6.6516 & 10.3575 & 10.338 \\
\hline 8.6000 & 4.3400 & 5.9816 & 0.9590 & 6.6930 & 6.7046 & 10.3216 & 10.3127 \\
\hline 8.4000 & 4.3680 & 6.0168 & 0.9461 & 6.7350 & 6.7519 & 10.3848 & 10.3752 \\
\hline 7.9000 & 4.2896 & 6.0520 & 0.5336 & 6.6604 & 6.6802 & 10.3416 & 10.3419 \\
\hline 7.7000 & 4.2560 & 5.8408 & 0.2213 & 6.5553 & 6.5702 & 10.0968 & 10.06 \\
\hline 8.8000 & 4.2280 & 6.2279 & 0.3803 & 6.6489 & 6.6614 & 10.4559 & 10.4265 \\
\hline 8.2000 & 4.3120 & 6.3335 & 0.3929 & 6.7752 & 6.7033 & 10.6455 & 10.6377 \\
\hline 8.1000 & 4.3120 & 6.2631 & 0.3510 & 6.7528 & 6.6965 & 10.5751 & 10.5711 \\
\hline 8.3000 & 4.2616 & 6.2983 & 0.3062 & 6.7084 & 6.7003 & 10.5599 & 10.5679 \\
\hline 7.8000 & 4.2308 & 5.8408 & 0.6753 & 6.5277 & 6.5363 & 10.0716 & 10.085 \\
\hline 7.7000 & 4.2336 & 5.9464 & 0.9913 & 6.5649 & 6.563 & 10.1800 & 10.1984 \\
\hline
\end{tabular}


Optimization of Cutoff Frequency Limits of Microstrip Circular Patch Antenna Printed on Ferrite ..

\begin{tabular}{|l|l|l|l|l|l|l|l|}
\hline 8.5000 & 4.2364 & 6.1223 & 0.3070 & 6.6245 & 6.6577 & 10.3587 & 10.3537 \\
\hline 8.4000 & 4.2700 & 6.0871 & 0.3078 & 6.6502 & 6.6607 & 10.3571 & 10.3526 \\
\hline 8.3000 & 4.3848 & 6.3335 & 0.5870 & 6.8555 & 6.8657 & 10.7183 & 10.6987 \\
\hline 7.8000 & 4.3540 & 6.1927 & 0.9566 & 6.7765 & 6.8032 & 10.5467 & 10.5338 \\
\hline 8.7000 & 4.6368 & 6.3335 & 0.6962 & 7.1321 & 7.1331 & 10.9703 & 10.8972 \\
\hline
\end{tabular}

\section{Conclusion}

The neural network is employed to optimize the cutoff frequency limits $\left(w_{l} \& w_{u}\right)$ of microstrip circular patch antenna printed on ferrite substrate and placed in perpendicular external magnetic field. In this communication for the design procedure, synthesis is defined as the forward side and then analysis as the reverse side of the problem. Here the analysis is considered as a final stage of the design procedure, therefore the parameters of the analysis ANN network are determined by the data obtained reversing the input-output data of the synthesis network. Thus, the extraordinary propagation constant $\left(\mathrm{K}_{\mathrm{e}}\right)$ resulted from the synthesized antenna geometry is examined against the target in the analysis ANN network.

\section{Acknowledgement}

The authors are grateful to Dr. R. Muralidharan, Director of "Solid State Physics Laboratory, Timarpur, Delhi" for providing necessary facilities, encouragement and motivation to carry out this work.

\section{References}

[1] D.M. Pozar' "Radiation and Scattering Characteristic of Microstrip Antenna on Normally Biased Ferrite Substrate", IEEE Trans., 1992, AP-40, pp.-1084-1092.

[2] L. Dixit, P.K.S Pourush, et. al. "Radiation characteristics of switchable ferrite microstrip array antenna", IEE Proc. Microw. Antennas Propag., Vol 147, No. 2, April 2000.

[3] N.K. Saxena, A. Khan, P.K.S Pourush and N. Kumar, "Neural Network Analysis of Switchability of Microstrip Rectangular Patch Antenna Printed on Ferrite Material", International Journal of RF and Microwave Computer-Aided Engineering, Vol. 20(1), pp. 1-5, 2009.

[4] S. Sa gıro glu, K. G"uney, M. Erler, "Resonant frequency calculation for circular microstrip antennas using artificial neural networks", International Journal of RF and Microwave Computer-Aided Engineering,Vol. 8, No. 3, pp. 270-277, 1998.

[5] D. Karabo ga, K. G"uney, S, . Sa garo glu, M. Erler, "Neural computation of resonant frequency of electrically thin and thick rectangular microstrip antennas", Microwaves, Antennas and Propagation, IEE Proceedings-Vol. 146, No. 2, pp. 155 - 159, April 1999.

[6] K. G"uney, S, . Sa giro glu, M. Erler, "Generalized neural method to determine resonant frequencies of various microstrip antennas", International Journal of RF and Microwave Computer-Aided Engineering, Vol. 12, No. 1, pp. 131-139, January 2002.

[7] P.K.S. Pourush and L. Dixit, "A 2x2 Element Planar Phased array of Circular Microstrip Antenna on Ni-Co Ferrite Substrate at 10 GHz", I.J. of Ratio \& Space Physics, 27, 289-226, 1998.

[8] P.K.S. Pourush et. al. "Microstrip Scanned Array Antenna on YIG Ferrite Substrate", Proc. International Symp. on Antennas and propagation, Japan 2000.

[9] Dheeraj Kumar and P.K.S. Pourush, "Circularly Polarized Microstrip Triangular Array on Normally Biased Yttrium Ferrite", Proceedings of Asia Pacific Microwave Conference, pp.856 Dec. 2004, New Delhi.

[10] C. A. Balanis, Antenna Theory, John Wiley \& Sons, Inc, 1997.

[11] M.S. Sodha and N.C. Srivastav's "Microwave Propagation in Ferrimagnetics" (1981), Plenum Press, New York.

[12] Q. J. Zhang, K. C. Gupta, Neural Networks for RF and Microwave Design, Artech House Publishers, 2000.

[13] J. Park, W. I. Sandberg, "Universal Approximation Using Radial Basis Function Networks", Neural Computation, Vol. 3, pp. 246$257,1991$. 